\title{
Evolución microestructural de capas delgadas de cobre por flujo de corriente eléctrica aplicada
}

\author{
P. QUINTANA*, A. I. OLIVA, F. ALONZO, J. E. CORONA, W. CAUICH \\ Cinvestav del IPN Unidad Mérida, Depto. de Física Aplicada, Km. 6 Antigua Carretera a Progreso, C.P. 97310, Mérida Yucatán, México.
}

\begin{abstract}
Se estudió la evolución microestructural superficial, durante el paso de corriente eléctrica, en capas delgadas de cobre. El análisis morfológico se realizó con microscopía de fuerza atómica (MFA), y la estructura por difracción de rayos X (DRX) con geometría de haz rasante, aplicando "in situ" un flujo de corriente DC entre 0 y 2.2A a través de electrodos colocados en los extremos de las capas. En muestras recién crecidas, se observaron picos de difracción desplazados a valores mayores de $2 \theta$ respecto del estándar de cobre, mismos que se acercan hacia el valor estándar conforme la corriente es incrementada. Se encontró que el flujo de corriente prolongado, así como los altos valores de corriente aplicada, favorecen el proceso de oxidación del cobre, formando cuprita $\left(\mathrm{Cu}_{2} \mathrm{O}\right)$. Morfológicamente se observó que el flujo de corriente modifica el tamaño del grano; mientras que el valor $r m s$ de la rugosidad, disminuye inicialmente para luego incrementarse debido a la formación del $\mathrm{Cu}_{2} \mathrm{O}$. La oxidación del cobre fue estudiada por espectroscopía Auger y por DRX.
\end{abstract}

Palabras clave: cobre, capas delgadas, DRX, MFA, espectroscopia Auger

\section{Microstructural evolution of copper thin films by applied electrical current flow}

The microstructural evolution during electrical current flow application on copper thin films was studied. The morphological analysis by atomic force microscopy (AFM), and the structural analysis by grazing incidence X-ray diffraction (XRD) were realized when an electrical current between 0 and 2.2A was applied in situ on films by means of electrodes located on films. Positions of peaks diffraction of the asdeposited film were found to be larger than the $2 \theta$ copper standard value, with a further decrement with the applied current. Cuprite $\left(\mathrm{Cu}_{2} \mathrm{O}\right)$ is formed when the current is hold for longer times and/or when high current values are applied, favouring the oxidation process. Morphological images show that grain size is modified when the current is applied; meanwhile the rms-rugosity, initially diminishes and after that, increases due to the high current values forming $\mathrm{Cu}_{2} \mathrm{O}$ on surface. Copper oxidation was studied by Auger spectroscopy and XRD.

Keywords: copper, thin films, XRD, AFM, Auger spectroscopy

\section{INTRODUCCIÓN}

Las interconexiones metálicas juegan un papel primordial en aplicaciones electrónicas, debido a la miniaturización de dispositivos, para incrementar la funcionalidad de los circuitos integrados, mejorar su desarrollo y disminuir los costos. Con los avances de la tecnología de los semiconductores desde los años sesenta hasta los noventa, se ha incrementado la demanda en el desarrollo y el conocimiento de las propiedades de las capas delgadas [1].

Se ha sugerido utilizar el $\mathrm{Cu}$ en las interconexiones eléctricas en circuitos integrados, en lugar del $\mathrm{Al}$ o sus aleaciones. Esto debido a la menor resistividad eléctrica del $\mathrm{Cu}(1.7 \mu \Omega$-cm) en comparación con el $\mathrm{Al}(2.7 \mu \Omega-\mathrm{cm})$, ya que mejora su desempeño eléctrico al reducirse el calentamiento por efecto Joule, logrando un transporte de calor más eficiente [2]. Por otro lado, dado que el punto de fusión del $\mathrm{Cu}$ duplica al del $\mathrm{Al}\left(1100\right.$ y $660^{\circ} \mathrm{C}$, respectivamente), disminuyen las fallas inducidas por electromigración debido a los procesos de difusión atómica y mejora sustancialmente la resistencia a la electromigración [3,4]. Sin embargo, el $\mathrm{Cu}$ en presencia de oxígeno muestra un proceso de corrosión, debido a que en forma pura no desarrolla una capa estable protectora de la oxidación, como sucede con el $\mathrm{Al}$, por lo que en la fabricación de circuitos integrados pueden generarse defectos superficiales. El papel que juega la microestructura en la electromigración del $\mathrm{Cu}$, es menos conocido que en el Al [5]. En el cobre se ha propuesto que la difusión superficial predomina sobre la difusión en las fronteras de granos [6]. Existen diversas propiedades que requieren ser estudiadas en el proceso de metalización del cobre: la electromigración, para determinar la cinética del crecimiento de defectos (agujeros) y los cambios en sus propiedades eléctricas en interconexiones [7]; los esfuerzos creados por efectos de migración [8]; las propiedades en la interfase capa-sustrato y los efectos dinámicos de transporte de masa que se generan por el paso de una corriente eléctrica en películas delgadas [9]. Se han realizado estudios de aleaciones de aluminio con bajas concentraciones de cobre, para analizar la formación de montañas y esfuerzos, debido a que presentan una baja resistividad eléctrica y una gran resistencia a la electromigración en comparación con las de Al puro [10]; sin embargo, muy pocos estudios sobre el efecto en la microestructura y cristalinidad de la superficie metálica al hacer pasar in situ un flujo de corriente existen en la literatura. El comportamiento microestructural del $\mathrm{Cu}$ es muy diferente al del $\mathrm{Al}$, por lo que es necesario profundizar en su evolución para optimizar ciertas características como la textura superficial, el tamaño de grano, etc. El recocido hasta $400^{\circ} \mathrm{C}$ origina un crecimiento del grano con la temperatura, donde la presencia de contaminantes o de una segunda fase en aleaciones muestra una fuerte influencia en el crecimiento de los granos [11].

En este trabajo se estudia la evolución microestructural superficial de capas delgadas de $\mathrm{Cu}$ depositadas sobre un sustrato rígido cuando se aplica in situ una corriente eléctrica. Los cambios en la rugosidad superficial y en la estructura cristalina fueron analizados por MFA y por DRX en haz rasante como función de la corriente DC aplicada. Se estudia el proceso de oxidación superficial usando espectroscopía Auger. 


\section{SISTEMA EXPERIMENTAL}

Usando evaporación térmica y Cu de alta pureza (99.999\% de Balzers), se depositaron capas delgadas sobre sustratos de vidrio Corning 7059 de $1 \mathrm{~mm}$ de espesor en el interior de una cámara evacuada con una bomba turbomolecular a una presión de $5 \times 10^{-6}$ Torr. La temperatura del sustrato se mantuvo a temperatura ambiente, con una distancia de separación de la fuente de $120 \mathrm{~mm}$. Se depositaron capas de geometría rectangular $(2 \times 10 \mathrm{~mm})$ con una velocidad de $2 \mathrm{nms}^{-1}$, con espesores entre 0.11 y $0.83 \mu \mathrm{m}$. Después del depósito, las muestras se mantuvieron dentro de la cámara durante dos horas, para asegurar que la temperatura de la capa fuera uniforme y evitar tensiones térmicas al abrirse a la presión atmosférica.

Para la aplicación de la corriente, se utilizó un portamuestra con electrodos esféricos sometidos a presión sobre la capa metálica y sobre los cuales se aplica el flujo de corriente DC con una fuente de poder HP-6224B. El intervalo de la corriente aplicada osciló entre 0 y $2.2 \mathrm{~A}$, con una densidad de corriente promedio de $1 \times 10^{4} \mathrm{~A} / \mathrm{cm}^{2}$, que es un valor menor comparado con el utilizado en estudios de electromigración y aplicaciones electrónicas [12]. Una vez colocada la muestra en el MFA, con el cantiliver retirado y sin mover la muestra del equipo, se hace pasar un valor de corriente durante una hora, se deja enfriar durante 10 minutos y se procede a la obtención de imágenes. Se utilizó un scanner de alta resolución (máximo 5 micras) y las imágenes fueron obtenidas a $1 \mathrm{~Hz}$ y con resolución de 256x256 pixeles ${ }^{2}$. Las mediciones topográficas se realizaron a temperatura ambiente y a presión atmosférica.

Los cambios estructurales se analizaron con una difractómetro Siemens (D5000) con radiación de $\mathrm{Cu}_{\alpha}(\lambda=1.5418 \AA ̊$ ). Los picos se registraron con paso de $0.01^{\circ}$ por cada 5 segundos, una apertura de la rejilla de divergencia de $0.1 \mathrm{~mm}$. El haz de rayos $\mathrm{X}$ incidió en forma rasante sobre la muestra con ángulos entre $0.4^{\circ}$ y $0.6^{\circ}$, dependiendo del espesor de la capa analizada. El análisis estructural se realizó durante la aplicación in situ del flujo de corriente; para ello, se utilizó un portamuestra diseñado especialmente para este fin [13]. Para el registro de los picos de difracción, se hace pasar previamente un flujo de corriente constante a través de la capa metálica durante una hora lo cual genera un calentamiento. Una vez estabilizada térmicamente la muestra, se procede a registrar el difractograma para el correspondiente valor de corriente aplicada. Es importante no mover la muestra de su posición durante la aplicación de todo el ciclo de corriente, para poder comparar entre sí los resultados. Un ciclo de medición equivale a elevar discretamente la corriente desde 0 hasta 2.2 A y luego disminuirla de nuevo a $0 \mathrm{~A}$.

La espectroscopia Auger utilizada para evaluar la capa de óxido formada en la superficie metálica se efectuó con un equipo PerkinElmer (PHI-560) con una presión de $5 \times 10^{-10}$ Torr. Se utilizó un haz de electrones primarios con $2 \mathrm{keV}$ y $0.02 \mathrm{~mA}$. La superficie de las capas fue erosionada con iones de Argón durante el análisis.

\section{RESULTADOS}

La superficie de las capas de cobre recién crecidas por evaporación, sobre sustratos de vidrio, es rugosa e inhomogénea con una estructura granular. Muestra grandes granos con diferentes alturas y geometría definida. El difractograma de la superficie metálica mostró una excelente orientación cristalográfica ya que la relación entre las intensidades de las difracciones principales de $I(111) / I(200)=6$, es casi tres veces mayor que el patrón del estándar del cobre policristalino; además, las reflexiones están ligeramente desplazadas hacia valores mayores de $2 \theta$, debido a los esfuerzos intrínsecos generados durante el crecimiento. Este comportamiento difiere de otros estudios, donde el cobre muestra una textura definida en el plano (200) con la presencia de cobalto [11]; sin embargo, es similar cuando el depósito se realiza por haz de iones asistido [14].

Para analizar la variación estructural de la capa metálica con la corriente, se registró el pico principal (111) por difracción de rayos $\mathrm{X}$ aplicando un flujo simultáneo de corriente para posteriormente ir incrementando el valor del mismo. Este proceso se repitió en varias capas y en todos los casos se halló el mismo comportamiento: los picos de difracción se desplazaron hacia valores menores de $2 \theta$, es decir, tendiendo hacia el estándar de cobre $\left(2 \theta=43.295^{\circ}\right)$. Los picos son definidos y estrechos por lo que mostraron una alta cristalinidad, con un tamaño promedio de cristalito de $64 \mathrm{~nm}$. No se observó un cambio significativo en su tamaño, aún manteniendo la muestra a 1 A durante $100 \mathrm{~h}$. El tamaño de cristalito es similar al obtenido cuando la película es depositada sobre sustratos de vidrio por otros métodos, aunque diferente cuando se deposita sobre Si [14]. La Figura 1 muestra un ligero incremento en los picos de difracción al incrementar paulatinamente el valor de la corriente, desde $0 \mathrm{~A}$ (denominada $0_{\mathrm{i}}$ ) hasta 1.4 A que fue la máxima corriente aplicada. La intensidad del pico (111) disminuyó hasta la mitad de su intensidad al disminuir la corriente y concluir el ciclo a $0 \mathrm{~A}$ (denominada $0_{\mathrm{f}}$ ), la posición final en $(2 \theta)$ alcanzó un valor ligeramente mayor que al inicio del experimento. Debido al calentamiento Joule de la muestra, al incrementar la corriente hasta $1.6 \mathrm{~A}$, se rompieron los contactos y se observó la aparición de unas manchas oscuras sobre la superficie de la capa de cobre. Estos resultados son similares a las aleaciones de cobre donde a bajas temperaturas $\left(<300^{\circ} \mathrm{C}\right)$ no se detectan cambios debido a esfuerzos ya que el coeficiente de expansión térmico permanece casi constante [11], a diferencia del aluminio que se comporta como un material elástico [15]. Este desplazamiento ocurre a bajas temperaturas $\left(120^{\circ} \mathrm{C}\right)$, por lo que el cobre se comporta como un material plástico bajo estas condiciones [16].

La Figura 2 (a y b) muestra imágenes AFM $\left(1 \times 1 \mu \mathrm{m}^{2}\right)$ de la superficie de cobre cuando se han aplicado diferentes flujos de corriente. La superficie recién depositada $(\mathrm{I}=0 \mathrm{~A})$ muestra granos alargados con un tamaño entre 0.05 y $0.2 \mu \mathrm{m}$. Al ir aumentando la corriente, la superficie se vuelve homogénea, con granos relativamente simétricos y cuyos diámetros disminuyen hasta $0.03 \mu \mathrm{m}$. Sin embargo, después de aplicar

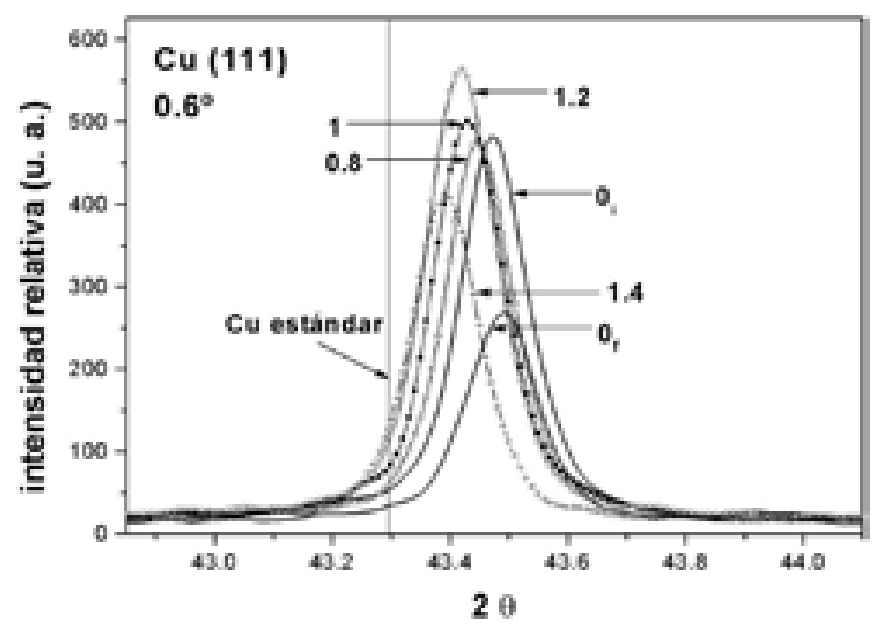

Figura 1. Evolución del plano (111) con un flujo in situ de corriente a través de una capa de cobre depositada sobre vidrio. El pico se registró a un ángulo rasante de $0.6^{\circ}$. 

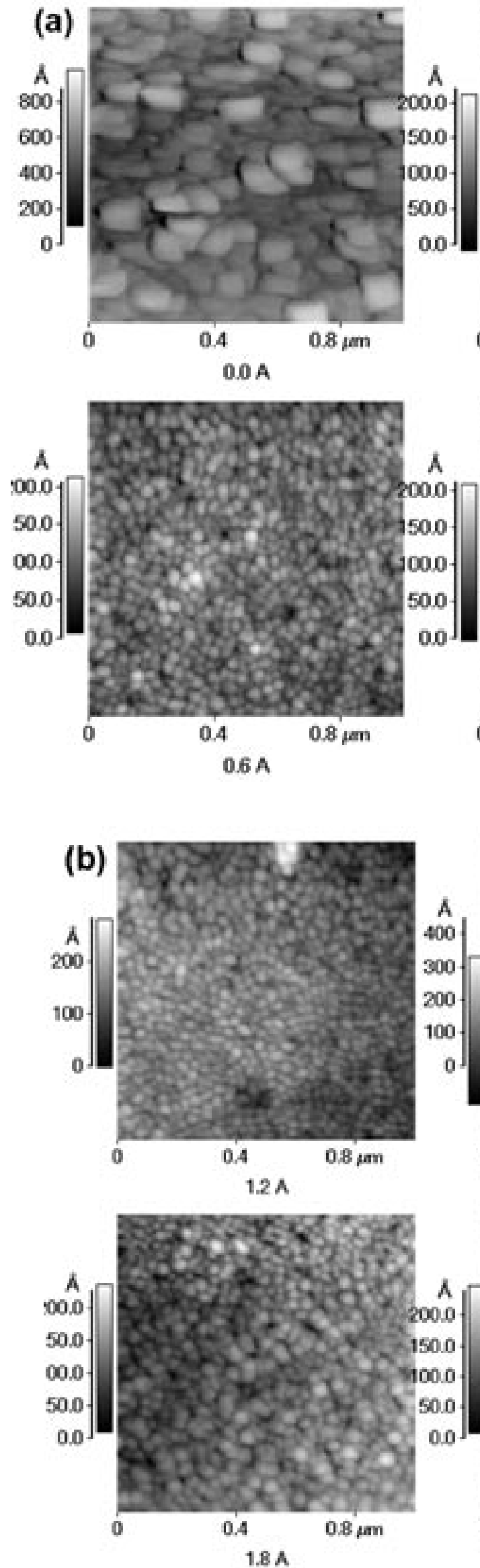
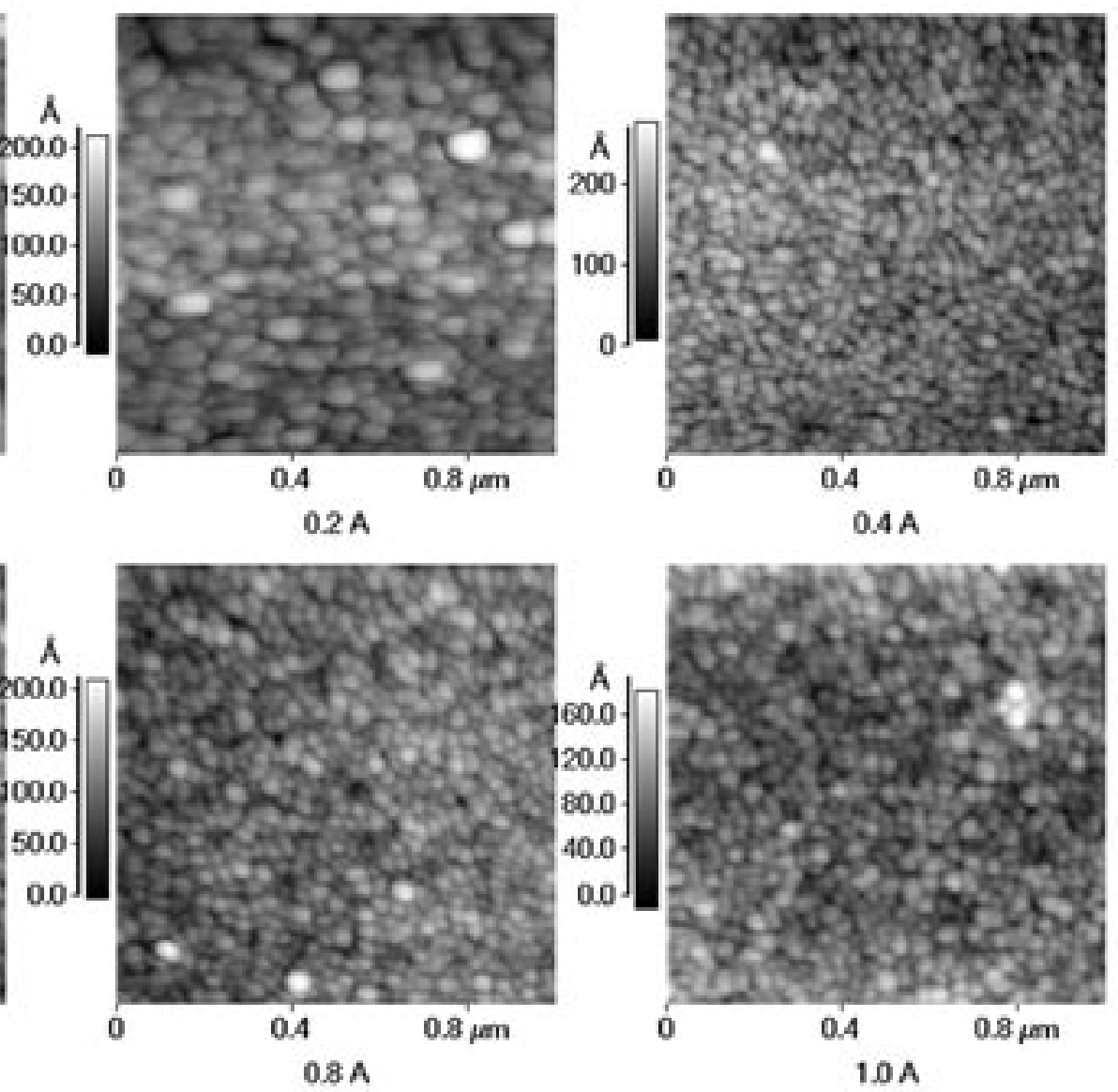
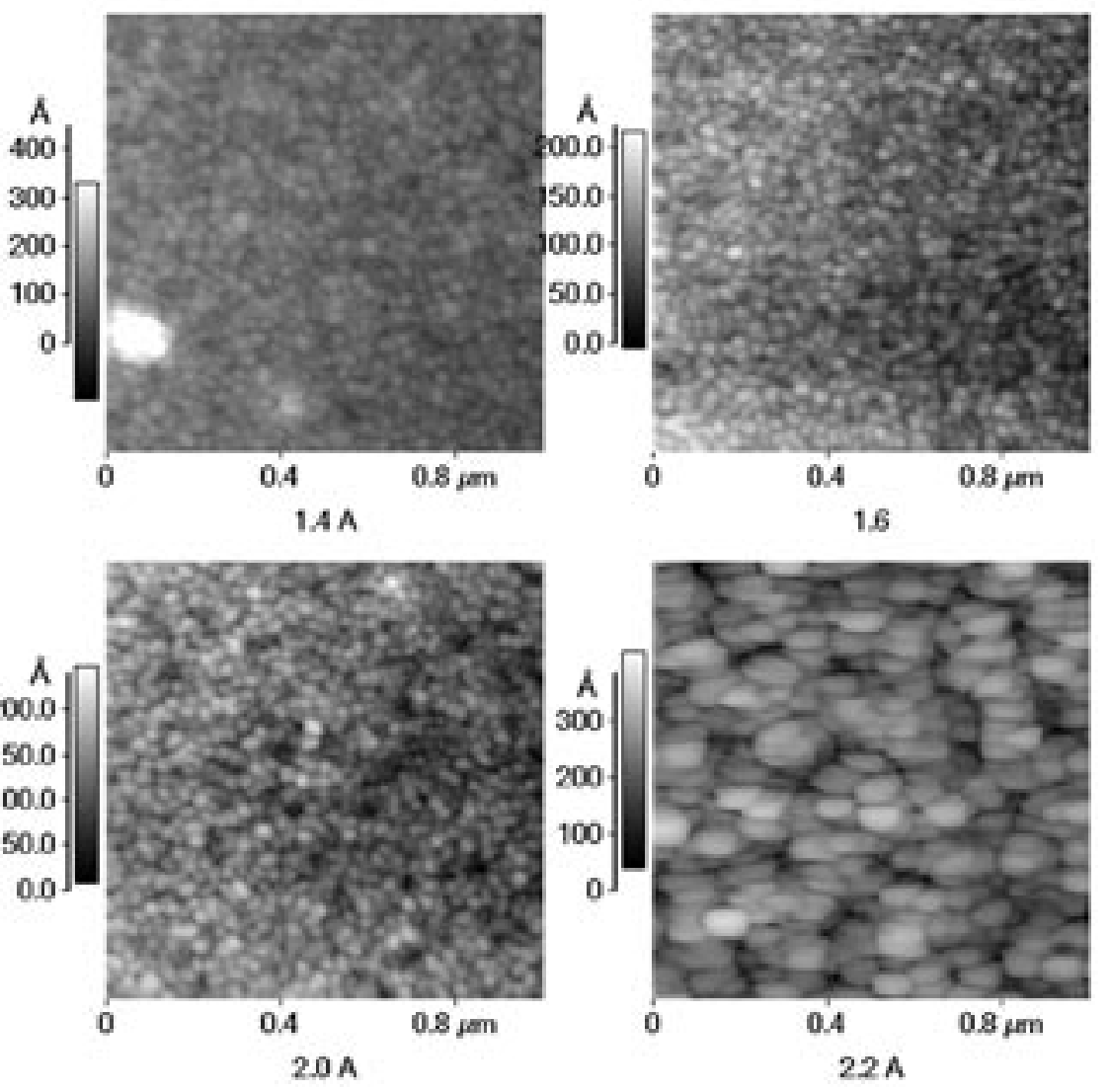

Figura 2. Imágenes AFM $\left(1 \times 1 \mu \mathrm{m}^{2}\right)$ de la superficie de cobre para diferentes valores de corriente aplicados. a) De 0 a $\left.1 \mathrm{~A} ; \mathrm{b}\right)$ de 1.2 a $2.2 \mathrm{~A}$. 


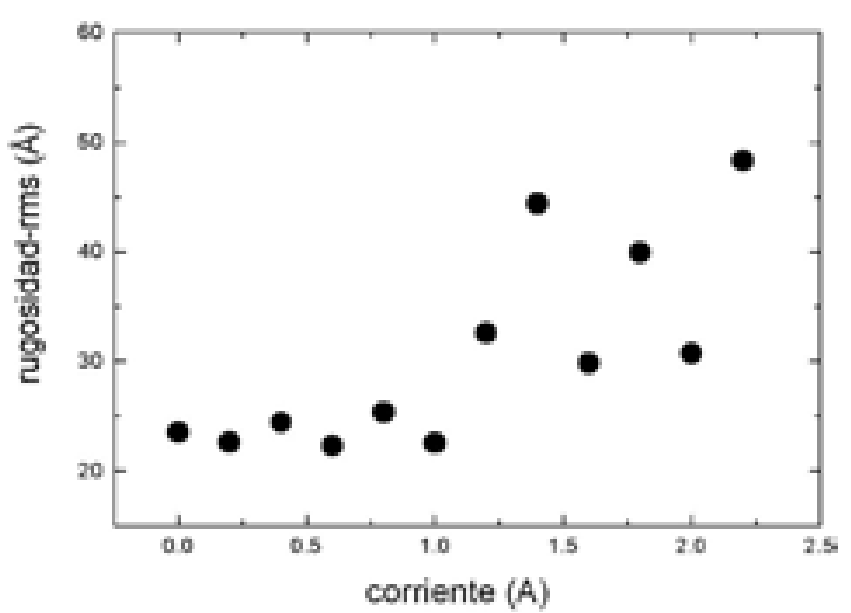

Figura 3. Valores rms de la rugosidad superficial correspondiente a las imágenes de la Figura 4.

1.6 A, se observa que los granos aumentan nuevamente y alcanzan tamaños similares a los iniciales. A cada imagen obtenida, se le calculó la rugosidad rms y se graficó contra el valor del flujo de corriente aplicado. En la Figura 3, se muestra la evolución de la rugosidad con la corriente aplicada con dos rangos de comportamiento: i) de 0 hasta valores de 1.0 A la rugosidad tiene un valor casi constante; ii) de 1.2 a 2.2 A se incrementa para valores de corriente elevados.

Por otro lado, las capas de Cu sufren un cambio de coloración superficial al aplicarles corrientes elevadas. Este mismo fenómeno se observó al aplicar corriente para efectuar las mediciones de rugosidad por MFA. Para conocer la razón de este cambio de color, se procedió a analizar las muestras mediante espectroscopia Auger. Se seleccionó en una misma muestra una zona que mostrara cambios en la coloración y se comparó con una zona sin cambios. En la zona no afectada, se detectaron principalmente concentraciones de $\mathrm{Cu}$, y en menor proporción $\mathrm{O}_{2}$, N y C, provenientes del efecto de oxidación en condiciones ambientales (Figura 4). Sin embargo, en la zona con cambio de coloración (alterada por la alta corriente), se observó un fuerte incremento en las señales características del oxígeno indicando que la superficie sufrió una fuerte oxidación proceso que fue favorecido por la temperatura.

Midiendo la intensidad pico-pico de las señales Auger como función del tiempo de erosión (10nm/min) para el $\mathrm{Cu}$ y el $\mathrm{O}_{2}$ y comparándola con las señales del Si (proveniente del sustrato), se obtuvo que después de $500 \mathrm{~s}$ de erosión, la proporción de oxígeno incrementó. De esta manera, es posible estimar el espesor de la capa de óxido que en este caso corresponde a un valor de $800 \AA ̊$ (Figura 5). En forma adicional se analizó la estructura de la capa de óxido, usando difracción de rayos $\mathrm{X}$, y se encontraron trazas del pico principal del óxido de $\mathrm{Cu}$, en forma de cuprita $\left(\mathrm{Cu}_{2} \mathrm{O}\right)$.

El patrón de difracción registrado después de pasar un flujo de corriente durante $14 \mathrm{~h}$, es similar al correspondiente del estándar policristalino [17]. Se obtuvo que la relación entre las intensidades de los picos principales $I(111) / I(200)$ es de 2.18. El Cu es muy susceptible a la oxidación y para poder ser utilizado en interconexiones es necesario protegerlo de la oxidación ya que la formación de cuprita degrada sus propiedades eléctricas y mecánicas. Trabajos preliminares han determinado que su oxidación depende de la orientación del plano cristalino, encontrando que en el plano (100) es 10 veces más rápida que en el plano (111) [18]. Las impurezas, tal como el $\mathrm{O}_{2}$ en los granos del $\mathrm{Cu}$, pueden actuar de tal manera que impidan la evolución microestructural.
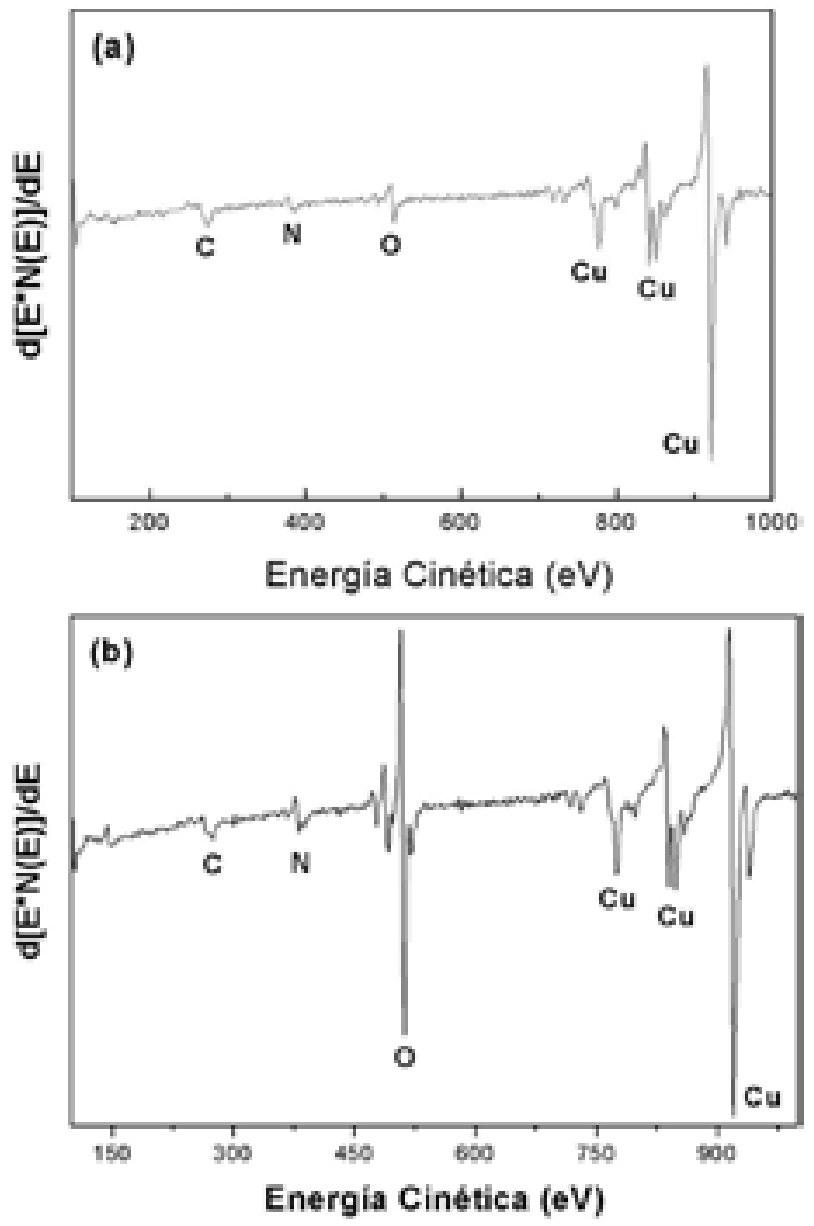

Figura 4. Análisis Auger de dos zonas de coloración diferente en la superficie del cobre. a) zona no afectada, b) zona afectada por el flujo de la corriente.



Figura 5. Superficie de Cu erosionada con Argón para medir la capa de óxido de cobre formada con la técnica Auger.

La rugosidad en las etapas iniciales, comparada con la capa recién depositada, disminuye drásticamente (Figura 3). Analizando cuidadosamente la imagen obtenida para $1.0 \mathrm{~A}$, se observa que los granos no muestran variación en el aspecto y la forma (Figura 2). Un efecto similar se ha observado en películas de oro depositadas sobre sustratos rígidos [19]. Por otro lado, a altos valores de corriente, la rugosidad se ve incrementada debido a la presencia y al crecimiento de la capa de óxido superficial. 


\section{CONCLUSIONES}

El flujo de corriente aplicado a las capas delgadas de $\mathrm{Cu}$ puro favorece la formación de su óxido en forma de cuprita. La morfología de la superficie observada por MFA y cuantificada en su valor $r m s$, se ve modificada y alterada. Esto implica que para poder utilizarse como conductor en un microdispositivo, es necesario adicionarle un recubrimiento de protección a la corrosión o agregarle un aleante que disminuya su degradación. Este efecto de degradación se ve favorecido con el incremento de corriente aplicada. La estructura cristalina de la capa delgada, no sufre cambios importantes, excepto por las tensiones generadas por la energía Joule involucrada. Sin embargo, el proceso simultáneo de la formación de la cuprita con la corriente aplicada, cuantificada por espectroscopia Auger, es una evidencia del proceso acelerado de degradación que pudiera presentarse bajo las condiciones experimentadas. A pesar de las excelentes propiedades eléctricas del $\mathrm{Cu}$, es necesario evitar su degradación para poder ser utilizado en la microelectrónica.

\section{AGRADECIMIENTOS}

Trabajo apoyado por el CONACYT (México) a través del proyecto No. 38480-E. Se agradece a D.H Aguilar y a Oscar Ceh por su ayuda técnica.

\section{REFERENCIAS}

1. M. Ohring, "Materials Science of Thin Films", Academic Press, New Jersey, 2002.

2. S.P. Murarka, I.V. Verner, R.J. Gutmann. “Copper-Fundamental Mechanisms for Microelectronic Applications", John Wiley \& Sons, N.Y, 2000.
3. J. R. Lloyd, J.J. Clement. "Electromigration in copper conductors" Thin Solid Films, 262 135-141 (1995).

4. C.-K. Hu, R. Rosenberg, K.Y.Lee. "Electromigration path in $\mathrm{Cu}$ thin-films lines". Appl. Phys. Lett., 74 2945-2947 (1999).

5. A.I. Oliva, P. Quintana, O. Ceh, J.E. Corona, M. Aguilar. “Current induced effects in aluminum thin films". Thin Solid Films 353 1-7 (1999).

6. C.S. Hau-Riege and C.V. Thompson. "Electromigration in Cu interconnects with very different grain structures". Appl. Phys. Lett., 78 3451-3453 (2001)

7. R. Frankovic and G.H. Bernstein. "Temperature dependence of electromigration threshold in Cu". J. Appl. Phys. 81 1604-1605 (1997)

8. Y.L. Shen, S.Suresh, M.Y. He, A.Bagchi, O.Kienznle, M.Rühle and A.G.Evans. J. "Stress evolution in passivated thin film of $\mathrm{Cu}$ on silica substrates". Mater. Res. 13 1928-1937 (1998).

9. S.P. Baker, A. Kretschmann and E. Arzt. "Thermomechanical behavior of different texture components in $\mathrm{Cu}$ thin films". Acta Mater. 49 2145-2160 (2001).

10. C. Kylner, L. Mattsson. "Initial hillock formation and changes in overall stress in Al-Cu films and pure Al films during heating". J. Mater. Res. 14 4087-4092 (1999).

11. J.M.E. Harper, E.G. Colgan, C.K. Hu, J.P. Hummel, L.P. Buchwalter and C.E. Uzoh. "Materials issues in copper interconnections". Mater. Res. Bull. 2923 29 (1994).

12. L.E. Levine, G. Reis, D.A. Smith. "In situ scanning-tunneling-microscopy studies of early-stage electromigration in Ag". Phys. Rev. B. 48 858-863 (1993).

13. J.E. Corona, D.H. Aguilar y P. Quintana. "Diseño y construcción de un portamuestras para analizar fenómenos de electromigración "in situ" por medio de difracción de rayos X con haz rasante". Congreso Nacional de Instrumentación SOMI XV, Guadalajara, Jal. México. Octubre 2000. Mat-4 p.1-6.

14. J. Yang, C. Wang, K. Tao, Y. Fan. "A new method to obtain Cu films with lower resistivity and higher interface adhesion on different substrates". J. Vac. Sci. Technol. A 13 481-484 (1995).

15. J.E. Corona, P. Quintana, D.H. Aguilar, A.I. Oliva, M. Aguilar. "Películas delgadas de aluminio: efectos inducidos por ciclos de corriente". Superficies y Vacío, 11 84-87 (2000).

16. M.D. Thouless, J.Gupta. J.M.E. Harper. "Stress development and relaxation in copper films during thermal cycling". J. Mater. Res. 8 1845-1852 (1993).

17. JCPDS-ICDD, Tarjeta No. 4-836 (2000).

18. J. Li and J.W. Mayer. "Refractory metal nitride encapsulation for copper wiring". Mater.Res. Bull. 28 52-56 (1993).

19. M. Aguilar, A.I. Oliva, P. Quintana. "Thickness stress relations in aluminum thin films". Mater. \& Manuf. Proc. 13 445-453 (1998). 